\title{
La causalidad estructural y la socionaturalidad de la depresión clínica: un tópico para la reflexión sobre la crisis de la salud pública
}

\section{Introducción}

El presente trabajo constituye un análisis teórico de la depresión, específicamente de los aspectos relativos a su causa y su naturaleza. Para llevar a cabo dicho análisis, se hizo una revisión conceptual, relativa al origen y naturaleza de la depresión, desde el punto de vista de la biomedicina, en la producción escrita más reciente de instituciones e investigadores de reconocido prestigio científico. Contando, de esta manera, con una visión general de la problemática actual relacionada con la compresión de esta enfermedad, así como de su tratamiento. Pero permitiendo, también, destacar que la evidencia científica apunta a que cada uno de los factores causa o etiológicos reconocidos así por la biomedicina, no son suficientes en sí mismos para que el fenómeno de la depresión se presente. Como instrumento de análisis, se recurrió al planteamiento de la causalidad estructural socionatural, interpretando, de acuerdo a sus conceptos, las explicaciones causales expuestas según la noción tradicional o biomédica. La aplicación de estos conceptos a la problemática de la depresión tiene el propósito fundamental de comprender integralmente su causa, de modo que contribuya, modestamente, tanto al logro de su explicación científica como a la orientación del desarrollo de acciones preventivas eficaces y de la atención integral de quienes la padecen.

\section{La depresión: definición y descripción}

La Clasificación Internacional de Enfermedades (CIE-10), en términos generales define a la depresión y a la manía como las tendencias que puede seguir 
una alteración del humor o de la afectividad, la cual se acompaña usualmente de una modificación en el nivel general de actividad, acorde a la tendencia del cambio del humor. El resto de los síntomas, que van a formar parte de la condición emergente, son mayoritariamente sistémicos a los cambios tanto del humor como de la actividad o fácilmente explicables por ellos. Estos trastornos son mayoritariamente de índole episódica, se inclinan a ser recurrentes y cada episodio suele ser precedido por sucesos estresantes (Organización Mundial de la Salud, 1992).

En términos particulares, señala que en los episodios depresivos típicos, el individuo sufre usualmente un decaimiento del ánimo, pérdida del interés y de la capacidad de disfrutar, con reducción de la energía que conduce a la fatigabilidad creciente y a la disminución de la actividad, y es común un cansancio marcado después de esfuerzos mínimos. Otros síntomas habituales son: a) reducción de la concentración y la atención, b) reducción de la autoestima y de la confianza en sí mismo, c) ideas de culpabilidad y de desvalorización, d) visión triste, sombría y pesimista del futuro, e) ideas o actos de autodaño o de suicidio, f) disturbio del sueño y g) disminución del apetito (ibid.).

Algunos de los síntomas antes mencionados pueden ser marcados y desarrollar características que se consideran extensamente como poseedoras de una significación clínica especial. Los ejemplos más típicos de estos síntomas llamados "somáticos" son: pérdida del interés o del placer en las actividades que son normalmente agradables; carencia de reactividad emocional al entorno y a los acontecimientos normalmente agradables; el despertar matinal dos horas o más antes de lo habitual; el empeoramiento de la depresión por las mañanas; evidencia objetiva de un definido retraso o agitación psicomotora (comentada o reportada por la gente); pérdida marcada del apetito; pérdida de peso (definida a menudo como $5 \%$ o más del peso corporal en el último mes); pérdida marcada de la libido. Generalmente, este síndrome somático no se considera como presente a menos que alrededor de cuatro de estos síntomas estén definitivamente presentes (ibid.).

El primer episodio puede presentarse a cualquier edad, entre la niñez y la senectud, su comienzo puede ser insidioso o abrupto, y su duración puede ir desde unas semanas a varios meses. Una duración de al menos dos semanas es usualmente requerida para su diagnóstico, pero períodos más cortos pueden ser razonables si los síntomas son inusualmente severos y de inicio rápido. El episodio depresivo puede ser calificado como leve, moderado o grave, su diferenciación descansa sobre un juicio clínico complicado que implica el número, el tipo y la severidad de los síntomas presentes (ibid.).

La depresión, como problema de salud, no es ajena a ningún grupo de edad, sexo o condición social. Sin embargo, es frecuente en adultos, presentándose alguna variación en cuanto a sexo, estado civil, ocupación y nivel socio econó- 
mico. No se registran variaciones significativas en cuanto al tiempo y lugar en que se presentan, alcanzando gran magnitud ya que su distribución es mundial (World Health Organization, 1997).

\section{Origen, naturaleza y manejo de la depresión según la noción tradicional}

Para establecer el pensamiento que actualmente predomina acerca de la naturaleza de la depresión y su origen, se ha decidido consignar la conceptualización que, al respecto, exponen fuentes con un alto prestigio académico y científico.

La Universidad de Harvard1' expresa que la depresión es una enfermedad de naturaleza y origen mental, y que es una enfermedad compleja con muy diversas causas. La depresión depende de la herencia, neuroquímica y enfermedades psiquiátricas o médicas, así como de la educación, las circunstancias sociales, las redes de apoyo (o la falta de apoyo), los acontecimientos que cambian nuestras vidas y las situaciones que creamos o que se encuentran dentro de nosotros mismos. De acuerdo con este planteamiento, casi todas las personas han tenido momentos de depresión y manía, pero estos humores pueden profundizarse y persistir cuando el equilibrio no se puede restaurar debido a un estrés inusual o a una regulación interna pobre. La falla del mecanismo regulador o el estrés excesivo pueden ser causados por susceptibilidad genética, desequilibrios bioquímicos, experiencias de la niñez y de la adultez, y circunstancias sociales, en proporciones que varían según diferencias individuales (Harvard College, 1998).

El neurobiólogo Charles B. Nemeroff ${ }^{2}$ manifiesta que psicólogos y neurobiólogos discuten a veces si las experiencias y los procesos psicológicos o biológicos causan la depresión. Sin embargo, la mente no existe sin el cerebro. Una evidencia considerable indica que sin importar los disparadores iniciales, las vías comunes finales a la depresión implican cambios bioquímicos en el cerebro. Son estos cambios los que dan lugar, en última instancia, a la tristeza profunda y a las otras características sobresalientes de la depresión. La acumulación extraordinaria de descubrimientos está aprovisionando un enérgico optimismo de que los principales determinantes biológicos de la depresión pueden ser entendidos detalladamente y que esas comprensiones abrirán el camino para mejorar los métodos de diagnosticar, de tratar y de prevenir la condición (Nemeroff, 1998).

El Instituto Nacional de Salud Mental (NIMH) ${ }^{3}$ afirma que la evidencia de la neurología, la genética y la investigación clínica demuestran que la depresión es un desorden del cerebro. Agrega que hay un alto grado de variación entre la gente con depresión en términos de síntomas, curso de la enfermedad y respuesta al tratamiento. Esta variabilidad no sólo plantea un desafío importante a los investigadores que procuran entender y tratar el desorden sino que también indica que la depresión puede tener un número complejo de causas que obran recíprocamente (National Institute of Mental Health, 1999). 
En particular, las causas a las que se atribuye la depresión, se exponen en los siguientes términos:

Los estudios de familia establecen que el índice de depresión en familias de pacientes deprimidos es 2 ó 3 veces mayor que el promedio y que el $25 \%$ de pacientes con depresión tienen un pariente con alguna forma de desorden afectivo. Esas familias también tienen un alto índice de otros desórdenes, tales como anorexia nerviosa y agorafobia con ataques de pánico (Harvard College, 1988).

Ahora, transmisión familiar no significa necesariamente transmisión genética. Sin embargo, los estudios de gemelos, una forma extensamente reconocida para hacer esta distinción, demuestran una vulnerabilidad hereditaria a la depresión.

Investigadores británicos, del Colegio de Medicina de la Universidad de Gales, examinaron la hipótesis de la heterogeneidad etiológica de la depresión. Es decir, la supuesta existencia de formas puras genéticas y no genéticas o, alternativamente, de una mezcla de ellas. Para ello eligieron 111 pares de gemelos (44 monocigóticos y 67 dicigóticos), de los cuales un gemelo, de cada par, había recibido tratamiento para la depresión, comprobado por vía del registro hospitalario. La información de los antecedentes familiares, sobre padres y hermanos, fue obtenida del sujeto, del co-gemelo o de ambos. El diagnóstico en padres y hermanos se hizo sin conocer el cigotismo del gemelo o concordancia y comparado en los parientes de los gemelos concordantes contra gemelos discordes. La prevalencia en el curso de la vida y el riesgo de depresión resultó ser similar en los parientes de pares de gemelos concordantes y discordes. A partir de lo cual concluyeron que los resultados constituian un argumento en contra del supuesto examinado y declaran que no es provechoso dividir la depresión en formas genéticas y no genéticas (Andrew, McGuffin y Katz, 1998).

A pesar de los múltiples y prolongados esfuerzos dedicados a buscar los genes asociados al trastorno, todavía no han podido ser identificados. Por lo que se piensa que la depresión es influenciada probablemente por varias combinaciones de genes que son diferentes en diversas familias y varían en sus efectos dependiendo del ambiente social y físico (Harvard College, 1998; Nemeroff, 1998; National Institute of Mental Health, 1999).

Las técnicas de exploración del cerebro, como la tomografía de emisión de positrones (PET) y la tomografía computarizada de emisión de fotones simple (SPECT), sugieren la interrupción o deterioro de las conexiones en las redes dedicadas a la regulación del humor. Los pacientes deprimidos (y posiblemente los vulnerables a la depresión) tienen una mayor actividad metabólica del cerebro total pero una actividad baja en la corteza prefrontal izquierda, que gobierna el juicio y el planeamiento. La amígdala, que regula la memoria para los acontecimientos emocionalmente significativos, está más activa de lo normal en la 
tristeza y la depresión clínica, y en pacientes que no están deprimidos ahora pero que lo han estado en el pasado (Harvard College, 1998).

Un hallazgo, que resulta coincidente con lo antes sugerido, es que la gente mayor seriamente deprimida tiene casi tantas anormalidades del cerebro como la que sufre demencia senil. En un estudio, que recurrió al uso de Imágenes de Resonancia Magnética (MRI), se compararon 67 pacientes hospitalizados con depresión severa recurrente (edad media 74) y 61 con demencia (edad media 75) con 44 controles mayores neurológicamente sanos (edad media 68). El 92\% de pacientes con demencia, el $85 \%$ de pacientes deprimidos y el $35 \%$ de los controles mostraron cierta anormalidad en la exploración del cerebro, generalmente atrofia de la corteza cerebral o leucoencefalopatía (degeneración de la sustancia blanca). Los pacientes deprimidos tenían más atrofia cortical que los controles y también más infartos (porciones de tejido fino que han muerto debido a fuente escasa de sangre), y sólo un índice mayor de atrofia cortical distinguió las exploraciones del cerebro de pacientes con demencia de las de pacientes deprimidos. Los investigadores concluyen que por lo menos en casos donde la enfermedad es tan severa que el paciente tiene que ser hospitalizado, la depresión en la gente mayor, como la demencia, está asociada a los cambios degenerativos del cerebro (Zubenko, Sullivan, Nelson et al., 1990).

El estudio de los neurotransmisores ha confirmado la defectuosa transmisión de señales en los circuitos implicados en la regulación del humor, los cuales descansan principalmente en los neurotransmisores monoaminos, norepinefrina y serotonina. A menudo, en los pacientes deprimidos, el sistema de suministro y distribución de norepinefrina y serotonina, no funciona apropiadamente. Especialmente cuando se está bajo estrés, demasiado o muy poco del neurotransmisor se libera a la hendidura sináptica, y los receptores en ambos lados responden demasiado débil o demasiado intensamente (Harvard College, 1998; Nemeroff, 1998). Esto se constata en el hecho de que la orina de muchos pacientes deprimidos tiene un nivel inusualmente alto o bajo de 3-metoxi-4-hidroxifenilglicol (MHPG), un producto de descomposición de la norepinefrina (Harvard College, 1988).

Es importante notar que la identificación de que la falla del sistema ocurre, sobre todo, ante la presencia del estrés, más concretamente ante la interacción del sistema regulador de pobre funcionamiento con el estrés, permite suponer que la deficiencia del sistema ya existía, lo cual sugiere que una condición. heredada podría estar expresándose en el desequilibrio químico.

Un hecho que contribuye a la corroboración de este aspecto es provisto por investigadores norteamericanos, del Departamento de Psiquiatría del Sistema de Cuidados de la Salud de New Jersey, al señalar que hay evidencia de que los factores genéticos se pueden incluir en los muchos determinantes del suicidio en la depresión. Ya que estudios de genética molecular han reportado polimorfismos 
en el gen hidroxilasa del triptófano que está implicado en la síntesis de serotonina. La susceptibilidad genética al suicidio en la depresión, sin embargo, tiende a afectar a individuos solamente en asociación con estrés o enfermedad psiquiátrica (Roy, Nielsen, Rylander, Sarchiapone y Segal, 1999).

El estudio del sistema endocrino ha comprobado la perturbación de la regulación de funciones corporales, altamente sensibles a los cambios del humor, que dependen de las secreciones hormonales suprarrenales y tiroidea. En pacientes deprimidos, las glándulas suprarrenales y tiroides no responden a las señales de disminuir o aumentar, respectivamente, la liberación de sus hormonas. El nivel en sangre de cortisol se mantiene alto -una de las más importantes hormonas esteroides secretadas por la corteza suprarrenal, la que entre sus muchas funciones incluye el control del balance de sal y agua, el metabolismo del azúcar, grasa y proteína, y la preparación del cuerpo y la mente para una emergencia. El nivel de tiroxina se mantiene bajo- hormona secretada por la tiroides, que influencia el crecimiento y el desarrollo sexual, y controla la tasa metabólica basal del cuerpo (Harvard College, 1998; Nemeroff, 1998; National Institute of Mental Health, 1999). Estos niveles sólo llegan a ser normales cuando los pacientes se recuperan (Harvard College, 1988).

La presencia de niveles hormonales anormalmente altos y bajos, se presenta a raíz del desequilibrio de los ejes hipotalámico-pituitario-adrenal (HPA) e hipotalámico-pituitario-tiroideo (HPT), llamados así porque la liberación de hormonas por parte de las glándulas endocrinas es gobernada por la glándula pituitaria, que a su vez recibe direcciones del hipotálamo. Ya que estos ejes mantienen su equilibrio por mensajes de retroalimentación, los que son a menudo difíciles de leer o de poca duración en la gente con depresión (Harvard College, 1998; Nemeroff, 1998; National Institute of Mental Health, 1999).

Los sistemas de neurotransmisores y endocrinos son interdependientes, y un desequilibrio de cualquiera de los sistemas altera la actividad de ambos. Un ejemplo de esta interdependencia, lo constituye el hecho de que la norepinefrina se produce por el cerebro y es secretada por las glándulas suprarrenales, por lo que también se le conoce como noradrenalina (Harvard College, 1998).

El estudio del ciclo del sueño ha evidenciado que los pacientes deprimidos pasan poco tiempo en las etapas III y IV, en las que el sueño se vuelve gradualmente más profundo. La etapa $\mathrm{V}$ o de movimientos oculares rápidos (REM), también conocida como sueño paradójico porque su actividad cerebral se asemeja al estado del despertar, generalmente comienza temprano en la noche y los sueños suelen ser inusualmente intensos (Harvard College, 1988, 1998).

Aunque las bases fisiológicas exactas del ciclo del sueño son desconocidas, se reconoce que su alteración no es ajena a los disturbios neuroquímicos y fisiológicos antes señalados, ya que, por lo menos en parte, este ciclo es gober- 
nado por vía cerebral usando norepinefrina y serotonina, y se liga a los ritmos de producción de cortisol (Harvard College, 1988).

La depresión coexiste a menudo con otros desórdenes psiquiátricos. Muchos pacientes esquizofrénicos están deprimidos crónicamente, y en la condición conocida como desorden esquizoafectivo, los síntomas de la esquizofrenia y de los desórdenes del humor aparecen íntimamente mezclados. Este diagnóstico presenta un problema teórico para la psiquiatría, desde que se piensa generalmente que la esquizofrenia y los desórdenes del humor tienen diversos orígenes biológicos (Harvard College, 1998).

El diagnóstico dual, abuso o dependencia de alcohol u otras sustancias y depresión, es una preocupación psiquiátrica cada vez más seria. Si el abuso de drogas causa depresión, la depresión conduce al abuso de drogas, o ambas tienen una causa común. En todo caso, una espiral viciosa sobreviene cuando los adictos utilizan sus drogas para aliviar los síntomas que las drogas han causado. La cocaína y otros estimulantes actúan directamente sobre los neurotransmisores en el cerebro causando euforia, que es seguida a menudo por depresión cuando cesa el efecto. Parece ser que la depresión en un adicto al alcohol o a la heroína a veces se vuelve notable después de la abstinencia (ibid.).

La mayoría de pacientes deprimidos muestra síntomas de ansiedad, y del $15 \%$ al $30 \%$ tiene ataques de pánico. En un estudio reciente de 2,000 pacientes diagnosticados con desórdenes de ansiedad, el $24 \%$ fueron rediagnosticados más adelante como deprimidos. Algunos de los síntomas y tratamientos de la ansiedad y de la depresión son similares, y pueden tener mecanismos subyacentes en común. Un diagnóstico propuesto de desorden mixto de ansiedad/depresión incluye síntomas físicos y emocionales de ambos (Harvard College, 1998; National Institute of Mental Health, 1999).

La gente con desórdenes de la personalidad -especialmente evasiva, dependiente, histriónica y limítrofe-- es altamente susceptible a la depresión, se desmoraliza más fácilmente por ella y su recuperación es más lenta. Se estima que la mitad de pacientes con desorden de personalidad limítrofe también sufren depresión. Algunos pacientes que, por su aburrimiento fácil, dramatizaciones e inestabilidad emocional, son diagnosticadas como personalidades limitrofes o histriónicas pueden estar sufriendo de un desorden a veces llamado depresión atípica. En teoría, la personalidad es más o menos duradera y penetrante, y los desórdenes del humor son episódicos, pero en la práctica no es fácil separar el tiempo emocional del clima emocional (Harvard College, 1988, 1998).

La depresión también coexiste frecuentemente con diversas enfermedades físicas. Las condiciones médicas crónicas asociadas a la depresión incluyen enfermedad cardiaca, cáncer, deficiencias vitamínicas, diabetes e infecciones tales como hepatitis y malaria. La depresión es también un efecto común de desórde- 
nes neurológicos, incluyendo las enfermedades de Parkinson y de Alzheimer, esclerosis múltiple, traumatismos y tumores del cerebro (Harvard College, 1998; National Institute of Mental Health, 1999). Un índice más alto de depresión se asocia particularmente a arteriosclerosis, ataques del corazón, tensión arterial alta, cáncer pancreático y esclerosis múltiple (Harvard College, 1998). Estos desórdenes pueden compartir algunos mecanismos biológicos subyacentes con la depresión (Harvard College, 1988).

La depresión es muy a menudo precedida por el estrés, que media un acontecimiento o situación que trastoma el equilibrio emocional o fisiológico y exige una adaptación difícil. Estos acontecimientos pueden ser diversos, tales como enfermedad, desempleo, accidentes graves, desastres naturales, muertes en la familia y separación de personas amadas. Pero también incluyen eventos que no son ordinariamente considerados como infortunios, tales como el matrimonio, el nacimiento de un hijo o la promoción a un trabajo con nuevas responsabilidades (Harvard College, 1988). Sin embargo, en la gente vulnerable, el riesgo de desarrollarla se considera mayor después de amenazas o de situaciones indeseables, particularmente la pérdida de una relación personal o de un vínculo social por muerte o separación (Harvard College, 1998; Nemeroff, 1998; National Institute of Mental Health, 1999). Se ha encontrado que durante los seis meses previos al comienzo de una depresión, los pacientes han experimentado más acontecimientos indeseables que en cualquier otro momento de sus vidas (Harvard College, 1988).

Ahora, es dudoso que alguna clase particular de acontecimientos esté relacionada específica o exclusivamente con la depresión. La misma decepción o amenaza conduce al aparecimiento de la depresión en una persona vulnerable, de la ansiedad en otra y de una psicosis aguda en una tercera. Del mismo modo, la depresión puede ser precedida por el divorcio, el desempleo o la enfermedad (Harvard College, 1998).

Finalmente, en la gente con desórdenes crónicos del humor, los episodios de depresión no tienen que ser necesariamente probables en los meses inmediatamente posteriores a los acontecimientos estresantes. Los acontecimientos de vida pueden contribuir a los episodios recurrentes de depresión en algunos individuos, mientras que en otros pueden presentarse sin disparadores identificables. La experiencia de la depresión por sí misma puede aumentar la sensibilidad del humor al estrés, posiblemente debido a cambios en el cerebro. Consecuentemente, menos estrés puede ser necesario para provocar cada episodio sucesivo (un fenómeno conocido como kindling) (Harvard College, 1998; National Institute of Mental Health, 1999).

Esto último es concordante con lo reportado por investigadores británicos, quienes buscaron determinar la sincronía de acontecimientos de vida, tales como conflictos maritales, desempleo, enfermedad y muerte de familiares, con episodios de depresión y manía. Durante un año, una vez cada dos meses, entrevista- 
ron a 51 pacientes que padecían crónicamente de desórdenes del humor $(24$ de depresión y 27 de desorden bipolar) y a 26 personas sin padecimiento alguno, con el propósito de registrar tanto la ocurrencia de acontecimientos que pudieran precipitar los episodios como los episodios mismos. La frecuencia de acontecimientos de vida en los seis meses anteriores a la primera entrevista era igual en los tres grupos. Los acontecimientos de vida no ocurrieron, en una frecuencia mayor al promedio antes establecido, en los dos meses previos a los episodios que presentaron los pacientes con desórdenes del humor, pero sí precedieron a las crisis emocionales que presentaron algunos de las personas sin ningún padecimiento psiquiátrico. Lo que llevó a concluir que, en pacientes con desórdenes crónicos del humor, sus episodios no suceden inmediatamente después de acontecimientos que alteran sus vidas (Pardoen, Bauwens, Dramaix et al., 1996).

En este mismo orden, existen diversas teorías psicológicas de la depresión, las cuales plantean sus explicaciones en las siguientes formas:

La teoría psicodinámica (psicoanalítica) ha sugerido que la depresión es una clase de duelo inconsciente. La gente deprimida ha perdido o fue privada de un padre u otra persona amada en la niñez, a veces por muerte o separación, más a menudo por el retiro del afecto. Pero no reconocieron conscientemente la pérdida y por ello el trabajo de duelo, un ajuste realista en vivir sin la persona amada, fue imposible. En su lugar, introyectaron (incorporaron dentro de sí mismas) la imagen de la persona perdida sin asimilarla. La libido (energía instintiva) retirada de la persona se dirige hacia la imagen introyectada en forma de cólera, que es reprimida. Más adelante, cualquier pérdida o decepción en la vida puede reactivar el duelo postergado y eventualmente sentido como depresión. La presencia de autorecriminación y culpabilidad, son expresión de cólera vuelta hacia el interior $u$ hostilidad inconsciente. Como la gente deprimida no sabe el significado de su dolor, porque la fuente y el objeto del duelo son inconscientes, es difícil disiparla, y sus síntomas persisten y vuelven (Harvard College, 1998).

La teoría del apego considera que los infantes que sufren la pérdida del vínculo emocional con sus madres, en un momento de la vida en el que necesitan estar cerca de ellas o de alguien equivalente para su seguridad y subsistencia, son vulnerables a la depresión. Este planteamiento, desarrollado en gran parte por el psiquiatra inglés John Bowlby, se sustenta en la investigación experimental de la respuesta a la pérdida de un vínculo emocional. Cuando los-monos infantes se apartan precipitadamente de sus madres, describen lo que se ha conocido como la respuesta de separación: primero protestan enojados como intento de encontrar y atraer la atención de sus madres, luego se desesperan y finalmente sobreviene la separación apática. Los niveles de cortisol se elevan en su sangre y también se altera la sensibilidad de sus receptores de neurotransmisores, de la misma forma que en los humanos deprimidos. Las observaciones de los niños que pasan por esta experiencia señalan que ellos tienden a volverse dema- 
siado inseguros para explorar el mundo por sí mismos. Es posible que estos niños sean menos capaces de hacer frente a las pérdidas como adultos y estén en peligro de desarrollar desórdenes del humor o desórdenes de la personalidad cuando adultos (ibid.).

La teoría conductista ve la depresión como una forma de comportamiento adquirido o aprendido. La gente que, por una u otra razón, recibe pocas recompensas tangibles $o$ intangibles por su actividad se vuelve socialmente aislada e inactiva, pues la falta de reforzamiento positivo da lugar a que una acción deje de repetirse. Si por ello recibe la recompensa de atención y condolencia, entonces se refuerza su pasividad. De seguir así, inadvertidamente se promueve tal comportamiento, la gente pierde interés, $y$ la espiral de inactividad y aislamiento social continúa profundizándose. Y los síntomas típicos de la depresión son el resultado. Una variante de esta teoría implica la idea de la indefensión aprendida. Cuando perros o ratas reciben reiteradamente descargas eléctricas inevitables, desarrollan síntomas físicos de depresión severa; sus movimientos se vuelven lentos, duermen y comen menos, y su crecimiento se detiene. También parece que pierden la esperanza de cambio y se vuelven totalmente pasivos, pues cuando están sujetos a otros choques que podrían prevenir, ni siquiera lo intentan. La teoría de la indefensión aprendida sugiere que la gente deprimida, como estos animales, ha perdido toda convicción de que puede cambiar las cosas porque ha estado sujeta tan prolongadamente a condiciones adversas más allá de su control (ibid.).

La teoría cognitiva enfatiza que los pensamientos son mantenidos también por el refuerzo y contribuyen a la depresión. La gente deprimida está convencida de que es indigna, de que no tiene valor, de que el mundo es hostil y de que el futuro no ofrece ninguna esperanza. Ante tal convencimiento, no se dan ningún crédito para el éxito, se culpan demasiado fácilmente cuando las cosas van mal, exageran la importancia de cada infortunio y subestiman el papel de lo accidental. Son vulnerables porque insisten en interpretar todo de modo pesimista. Ellos piensan de esa manera debido a "esquemas" latentes o presunciones no reconocidas sobre el significado de la experiencia que se aprenden temprano en la vida y son puestas rápidamente en juego ante cualquier decepción, pérdida o rechazo. Sin embargo, los pensamientos de autoderrota son sólo síntomas de depresión, cuando se convierten en parte de un ciclo destructivo en el cual la apatía, la tristeza y el retraimiento social se toman como muestras del fracaso, y esta respuesta intensifica la fatiga, la miseria y el aislamiento (ibid.).

Investigadores canadienses, del Departamento de Psicología de la Universidad McGill, examinaron la presencia de actitudes disfuncionales en la depresión, para establecer si estos factores son concomitantes estado-dependientes de este problema de salud o tienen una estabilidad relativa. Para ello se utilizaron datos del Programa de Investigación para la Colaboración al Tratamiento de la Depre- 
sión, del Instituto Nacional de Salud Mental, consistentes en calificaciones de la Escala de Actitudes Disfuncionales (DAS), previas y posteriores al tratamiento de la depresión. Los puntajes promedio disminuyeron después de 16 semanas de tratamiento, en la DAS total y particularmente en perfeccionismo y necesidad de aprobación. Sin embargo, las correlaciones test-retest demostraron que las variables de la DAS exhibieron una estabilidad relativa considerable. Los modelos de ecuación estructural demostraron que las actitudes disfuncionales después del tratamiento fueron significativamente predecibles por el nivel inicial de actitudes disfuncionales, así como por la depresión postratamiento. La estabilidad relativa de actitudes disfuncionales era incluso mayor durante el período de 18 meses posteriores. Los resultados fueron consistentes con lo planteado acerca de la vulnerabilidad por los principales expositores de la teoría cognitiva (Zuroff, Blatt, Sanislow, Bondi y Pilkonis, 1999).

Lo relativo al tratamiento de la depresión, tiende a ser expuesto en los mismos términos biomédicos en los cuales se la conceptualiza.

Los tratamientos más extensamente usados son los muchos antidepresivos y estabilizadores del humor introducidos en los últimos 40 años. La mayoría de los pacientes conseguirán cierto alivio a partir de uno de estos medicamentos o de una combinación de ellos. Todos han probado, más o menos, ser igualmente eficaces en pruebas controladas: de $65 \%$ a $85 \%$ de pacientes mejoran, comparado con la mejoría de $25 \%$ a $40 \%$ de pacientes que toman un placebo. La mayoría de los expertos piensan que los antidepresivos se deben continuar por meses o aún años después de la recuperación para prevenir recaídas. Muchos pacientes han tomado continuamente los medicamentos por 15 años o más. Casi todos los antidepresivos influencian la actividad de los neurotransmisores monoaminos y toman varias semanas para comenzar a trabajar (Harvard College, 1998; Nemeroff, 1998; National Institute of Mental Health, 1999).

La clase más antigua de antidepresivos son los tricíclicos (TCAs), que trabajan principalmente realzando los efectos de la norepinefrina -a través de retrasar su reabsorción y modificar la sensibilidad de sus receptores. Los tricíclicos, después de muchos años como tratamiento dominante, están siendo sustituidos debido a sus efectos secundarios: boca seca, visión borrosa, vértigos, somnolencia, aumento de peso, estreñimiento y dificultad en orinar- que resultan sobre todo de su influencia en la acetilcolina, un neurotransmisor no-monoamino. También representan un peligro de suicidio, ya que el suministro de dos semanas puede ser fatal si es tomado todo de una vez (Harvard College, 1998; Nemeroff, 1998; National Institute of Mental Health, 1999).

Un segundo grupo de antidepresivos son los inhibidores de la monoaminoxidasa (MAOIs), que realzan la actividad de la norepinefrina y la serotonina -interrumpiendo la acción de una enzima que cataliza su rompimiento. Son útiles para algunos pacientes que no responden a otros medicamentos, especial- 
mente los que sufren de depresión atípica o ataques de pánico. Los inhibidores de la monoaminoxidasa pueden causar vértigos, insomnio e impotencia, y pueden producir peligrosamente alta tensión arterial cuando son tomados con alimento o bebidas que contienen la sustancia tiramine (Harvard College, 1998; Nemeroff, 1998; National Institute of Mental Health, 1999).

Hoy en día, sin embargo, la primera opción para la mayoría de los pacientes deprimidos son los inhibidores selectivos de la recaptación de serotonina (SSRIs), que realzan la actividad de la serotonina a través de retrasar su reabsorción. Los inhibidores de la recaptación de serotonina tienen pocos efectos secundarios y no representan casi ningún peligro de suicidio por sobredosis. Pueden causar insomnio, agitación o reducción de la respuesta sexual, y, por la afectación de la actividad de enzimas del hígado, pueden retardar la eliminación de antihistamínicos, analgésicos y antidepresivos tricíclicos del cuerpo. Si se toman junto con inhibidores de la monoaminoxidasa o litio, puede resultar una reacción llamada síndrome de serotonina, cuyos síntomas incluyen taquicardia, tensión arterial alta y elevación de la temperatura corporal (Harvard College, 1998; Nemeroff, 1998; National Institute of Mental Health, 1999).

Y la novedad del momento, la constituye un remedio herbario conocido como el mosto de San Juan. La hierba se vende en almacenes de alimentos naturales en la forma de un extracto líquido o de cápsulas que contienen las flores y las hojas machacadas de la planta. Su ingrediente activo es al parecer una sustancia llamada hipericin, que realza la actividad de la serotonina y puede ser un inhibidor de la monoaminoxidasa. El mosto de San Juan ha probado ser tan eficaz como los antidepresivos tricíclicos, por lo menos por breves períodos, en varios estudios sin un control placebo, pero no se puede llegar a ninguna conclusión clara sobre su valor debido a una respuesta inusualmente alta a los placebos en otros estudios comparativos. Los efectos secundarios inmediatos de la hierba son de menor importancia, pero poco se sabe sobre riesgos a largo plazo (Harvard College, 1998; National Institute of Mental Health, 1999).

La terapia electroconvulsiva (ECT) es más eficaz que la mayoría de los tratamientos para la depresión, aunque es la más estigmatizada. Del $80 \%$ al $90 \%$ de gente con depresión severa mejora con este tratamiento. La terapia electroconvulsiva implica producir una dramática aprehensión en el cerebro de un paciente bajo anestesia general, aplicando un estímulo eléctrico al cerebro a través de electrodos colocados en el cuero cabelludo. Los tratamientos repetidos son necesarios para alcanzar la respuesta más completa de los antidepresivos. La pérdida de memoria y otros problemas cognoscitivos son consecuencias comunes del tratamiento, con todo los efectos secundarios son típicamente de breve duración. Aunque alguna gente manifiesta dificultades duraderas, los avances modernos en técnica de terapia electroconvulsiva han reducido grandemente los efectos secundarios de este tratamiento en comparación a décadas anteriores. La 
investigación ha encontrado que la dosis de electricidad aplicada y la colocación de los electrodos (unilaterales o bilaterales) pueden influir el grado de alivio de la depresión y la severidad de los efectos secundarios (National Institute of Mental Health, 1999).

Finalmente, también se recurre a la psicoterapia en la medida que constituye un recurso de modificación de la actividad cerebral. Como el proceso de aprender implica la formación de nuevas conexiones entre las células nerviosas en el cerebro, la psicoterapia trabaja cambiando la manera en que funciona el cerebro. $\mathrm{La}$ investigación ha demostrado que ciertos tipos de psicoterapia, particularmente la terapia cognoscitivo-conductual (CBT) y la terapia interpersonal (IPT), pueden ayudar a aliviar la depresión. Los pacientes que son ayudados con terapia cognitiva cambian los estilos negativos de pensamiento y de comportamiento asociados a menudo a la depresión. La terapia interpersonal se centra en el trabajo con las relaciones personales disfuncionales que pueden contribuir a la depresión (ibid.).

\section{El problema con la noción tradicional sobre el origen y naturaleza de la depresión.}

El estudio de diversos aspectos de la depresión, desde el punto de vista tradicional, ha demostrado que en su origen están involucrados una variedad de eventos -constitucionales, psicológicos y sociales- y que su presencia, en el individuo particular, es la expresión concreta de la alteración de mecanismos "biológicos" subyacentes. Estos resultados constituyen el fundamento sobre el cual se asienta la noción biomédica de su multicausalidad y de su naturaleza orgánica.

La inconveniencia, sin embargo, con estos conceptos es ¿qué se dice específicamente y qué se debe entender exactamente?. Cuándo se expresa que la depresión es una enfermedad con múltiples causas, ¿es el resultado de cualquiera de ellas, de a veces unas y a veces de otras, o de la acción del conjunto?. Cuándo se afirma que la depresión puede tener un número complejo de causas que obran recíprocamente, ¿cómo se articula esa acción?. Cuándo se manifiesta que la mente no existe sin el cerebro, ¿el cerebro es el origen de lo mental o el cerebro es la estructura en la que lo mental se cristaliza?. Cuando se manifiesta que al final son los cambios bioquímicos los que dan lugar a la depresión y cuando se afirma que la depresión es un desorden del cerebro, ¿lo psicológico y lo social son irrelevantes en su determinación o son relevantes pero lo biológico lo es aún más al grado que ello define su carácter?.

El punto es que, al recurrir a estos conceptos, no es posible explicar de una manera exacta por qué y cómo enfermamos de depresión. Y ante estas circunstancias, es necesario preguntarse ¿cuál es el resultado de esto?, ¿qué se deriva de 
una acción dirigida a evitar un problema de salud cuyo origen no se conoce con certeza? y ¿en qué medida es eficaz, eficiente y efectiva una acción encaminada a tratar un problema de salud que no se comprende completamente?, la respuesta no tiene muchas opciones y lo más probable es que el resultado es el aseguramiento de la presencia del trastomo.

Hay un problema, entonces, con la conceptualización de la depresión, en lo relativo a su causa y su naturaleza, en tanto que no constituyen una forma apropiada para explicarla integralmente y ello puede conllevar el déficit de la capacidad para proteger a la población de este trastorno y para brindar la atención integral que requieren quienes ya la padecen.

Sería erróneo, sin embargo, creer que esta situación sólo atañe al problema de salud en cuestión, incluso pudiera pensarse que este fenómeno es exclusivo de los problemas de salud mental, debido a su peculiaridad, y que la situación es distinta y más promisoria para los llamados problemas de salud física. Pero no parece ser así, hay razones para considerar que esta situación es, realmente, la expresión de un problema más fundamental.

A favor de esta aseveración, se encuentra el reconocimiento explícito, por parte de funcionarios relacionados con los organismos rectores de la salud en la región y en el mundo, de que la salud pública se encuentra inmersa en una crisis.

En una publicación de la Organización Panamericana de la Salud (OPS), que compila artículos dedicados al tema de la crisis de la salud pública, J. R. Ferreira, Coordinador del Programa de Desarrollo de Recursos Humanos de dicho organismo en ese momento, inicia su exposición diciendo: "En los últimos años se ha incrementado la conciencia sobre la crisis actual de la salud pública, entendida ésta como la incapacidad de la mayoría de sociedades de promover y proteger su salud en la medida en que sus circunstancias históricas lo requieren" (Ferreira, 1992: p. 233).

La explicación de las raíces de esta crisis aún continúa siendo una tarea pendiente. Sin embargo, las nociones predominantes acerca de la salud, de la enfermedad y del vínculo entre ellas, así como de lo que le da origen a ambas, son aspectos que seguramente desempeñan un papel fundamental.

La elaboración teórica, por parte de las ciencias de la salud, en torno a los conceptos de salud y de enfermedad, comprendidas como condiciones peculiares o como elementos que estructuran un proceso único, ha sido desigual.

La enfermedad, definida de acuerdo al pensamiento médico, es un estado dinámico caracterizado por ciertas alteraciones corporales y, en términos técnicos, representa una condición médicamente definida que es identificable en varias personas, la cual supone una causa desencadenante, un curso predecible y 
autonomía, es decir, que continúa su desarrollo a pesar del deseo del afectado por superarlo (Selva Sutter, 1990). La salud, en la mayoría de los casos, resulta ser un término sin definición propia y el concepto que se le asigna es el de noenfermedad, de normalidad o bienestar (Alarcón y Kroeger, 1991).

Lo anterior se cristaliza en la acción encaminada a eliminar la enfermedad con la pretensión de obtener la salud. Una práctica común a la que subyace la idea de que la salud y la enfermedad son condiciones absolutas que se alternan mutuamente.

Esa noción es falsa. La ausencia de enfermedad no supone necesariamente la presencia de salud. Una evidencia muy apropiada la constituye el fenómeno de la infección subclínica o asintomática, la cual, aunque no lleva al aparecimiento de signos y síntomas manifiestos (Benenson, 1987), exige el reconocimiento de que no es la salud la presente.

Ahora, esto no sólo constituye un problema científico sino que también constituye una injusticia social, porque un quehacer encaminado a la eliminación de la enfermedad produce, en el mejor de los casos, justamente eso, la ausencia de enfermedad y no necesariamente el logro de la salud.

George Alleyne, en su discurso al asumir el cargo de Director de la OPS en 1995, expresó: "Los países miembros [de la OPS] recalcan con orgullo que se ha observado un mejoramiento constante en sus indicadores de salud. Pero cualquier asomo de arrogancia es sofocado de inmediato por un hecho innegable que nos perturba: el número de pobres y de desposeídos aumenta y se ensancha la brecha entre los que nunca antes vivieron mejor y los que se preguntan si podrán siquiera sobrevivir. El reconocimiento de esta brecha que se ha convertido en un fenómeno mundial, llevó a que, en 1978, las naciones de todo el mundo, bajo la égida de la Organización Mundial de la Salud, enarbolaran el lema de la Salud para Todos como un vivo grito de justicia social y de equidad en la distribución de los recursos". Más adelante declaró: "Este es el trabajo, este es el cometido: escuchar y atender el clamor de todos aquellos que exigen salud ahora y siempre". Y agregó: "Nuestro primer cometido es dejar claro a todos que si nos concentramos única y exclusivamente en una visión estrecha de lo que debe ser la salud, y no vemos más que las causas de la enfermedad, estaremos cometiendo una injusticia con nuestros pueblos. Debemos abjurar para siempre el concepto mecanicista de la salud desprendido del reduccionismo, que dio buen resultado a Descartes y a Newton pero que ya no surte efecto. Tenemos que reiterar una y otra vez que la búsqueda de la salud es simplemente otro aspecto de la eterna lucha por el desarrollo humano" (Alleyne, 1995: p. 4).

De ahí que la escasa dedicación a pensar sobre la salud, que contrasta con el esmero puesto en la dilucidación del origen de la enfermedad, sin que esto último sea en sí mismo perjudicial, y la inexacta apreciación de la relación que 
la salud y la enfermedad guardan son, sin duda, algunos de los determinantes de la crisis aducida. Crisis misma que, a su vez, se constituye inevitablemente en uno de los determinantes de la enfermedad, ya que ante la imposibilidad de asegurar la salud se cede espacio a la enfermedad.

Un problema fundamental, entonces, es que la salud ha carecido de identidad, cuyas consecuencias han sido francamente desventajosas para la ciencia y para la sociedad.

Otro problema fundamental, lo es el atribuir a un factor o a múltiples factores, total o relativamente independientes entre sí, la causación de la enfermedad, pues la explicación de ésta resulta estrecha e imprecisa, limitando la comprensión del evento y la realidad objetiva que ello encierra.

Esto tiene que ver con el hecho de que en las explicaciones sobre el aparecimiento de la enfermedad, han imperado los modos de pensar propios de la medicina, las cuales se han apegado estrictamente al biologismo que predomina en esta ciencia, limitando el concurso de otras ciencias igualmente importantes y necesarias para la consecución de un modo de pensar íntegro.

El biologismo es el rasgo estructural del modelo médico hegemónico en la salud pública, que subordina en términos metodológicos e ideológicos a los otros niveles explicativos posibles. Lo manifiesto de la enfermedad es ponderado en función de este rasgo como lo causal, sin considerar a la red de relaciones sociales que determinan lo fenoménico de la enfermedad (Ferreira, 1992). Para la práctica médica, la enfermedad es, en primer lugar, un hecho natural, biológico, y no un hecho social histórico. Este enfoque ahistórico evita o limita observar la importancia de los procesos no biológicos, pues ignora los procesos histórico sociales que operan sobre el proceso salud/enfermedad (Najera, 1992).

En el modelo biomédico, la causa, a veces catalogada como factor-causa y a veces como factor de riesgo, es considerada tanto un factor independiente como un conjunto de factores [orgánico/biológicos, ambientales y psicosociales]. Pero aún en este último caso casi siempre se destaca a uno [lo biológico] como el principal y a los otros [ambientales y psicosociales] como predisponentes o ayudadores (García, 1994). Esta forma de interpretación, da lugar a constatar que el multicausalismo, en esencia, no es más que una sofisticación del monocausalismo. Pues aun cuando se considera a factores biológicos, psicológicos y sociales en la causa de los problemas de salud, se le adjudica principalidad a lo biológico, limitando u ocultando la participación del resto de los factores (Selva Sutter, 1990).

El problema de fondo, pues, es que el modelo biomédico dominante no permite la compresión integral del proceso salud/enfermedad y, por consiguiente, tampoco su explicación causal completa. La existencia de este hecho, demanda la necesidad de reflexionarlo con detenimiento y es propicio para confrontar el 
quehacer, y el pensamiento que lo guía, en relación con la salud y la enfermedad. Dos conclusiones que de inmediato pueden extraerse son, que la salud aún está lejos de ser una necesidad satisfecha, por tanto, la enfermedad está ganando la partida, y que el pensamiento con el que se enfocan la salud y la enfermedad es inadecuado.

\section{Aplicación del concepto de la causalidad estructural y de la socionaturalidad a la depresión}

Sagatovski y Antipov (1966), haciendo un replanteamiento filosófico general, apoyados en la concepción materialista dialéctica moderna, someten a análisis los conceptos de causa, condición, etiología y patogenia.

Plantean que este enfoque se apega al concepto corriente de causa, definido como un fenómeno que necesariamente provoca otro fenómeno, el cual es su consecuencia o su efecto. Pero puntualizan que tal causa sólo responde a esa definición, de provocación necesaria, si constituye la unidad de todas las condiciones internas y externas, y es simultánea con su efecto o consecuencia.

Las condiciones, referidas, comprenden a todos los fenómenos, internos y externos, relacionados con el efecto, por lo que todas estas condiciones son necesarias para que tal efecto se produzca. Entre las condiciones necesarias, ninguna puede considerarse absolutamente principal respecto al resto $y$, más aún, ninguna es una causa en sí misma pues ninguna es suficiente por sí misma para provocar el efecto.

Diferencian dos momentos en la composición de la causa. Emplean el término causa genética para referirse al conglomerado de condiciones necesarias que precede al efecto, pero que es insuficiente para provocarlo. Y el término causa estructural para referirse a un nuevo conglomerado de condiciones, que se conforma cuando al primer conglomerado aludido se agrega otra condición necesaria, y la presencia de ésta lo hace suficiente para provocar el efecto, que se produce simultáneamente ante su presencia.

La causa estructural es el conglomerado de condiciones interactuantes [o la interacción del conglomerado de condiciones], necesarias y suficientes para producir un fenómeno. En este conglomerado, el número de tales condiciones es muy definido $\mathrm{y}$, aunque naturalmente son muchas, ellas actúan como un todo o como un mecanismo único.

Si la causa estructural está presente, entonces su acción o su efecto estará presente. El que la causa sea simultánea con su efecto es una condición para preservar el carácter necesario de la relación causa/efecto. En este sentido, la causa y el efecto constituyen dos aspectos diferentes de un mismo proceso.

La causa estructural [en tanto interacción] es [en esencia] el mecanismo intemo de un fenómeno [en la provocación de otro fenómeno] y su efecto, el 
indicador que revela la existencia del mecanismo dado. Por tanto, descubrir la interacción [o la causa] significa conocer los componentes de la interacción y mostrar el mecanismo de esa interacción, así como de su resultado.

Esta interpretación de la causalidad es aplicada a los conceptos de etiología y patogénesis. La etiología se constituye por las condiciones que componen la causa estructural y cuya interacción provoca un efecto. La patogénesis es el mecanismo de esa interacción, y por ello es la causa estructural del surgimiento, curso y funcionamiento de un proceso observado.

Selva Sutter (1999), analizando el planteamiento original de los autores antes mencionados, expone una ampliación en algunos de sus conceptos y propone una forma de comprensión distinta y más precisa en otros.

Agrega conceptos acerca del carácter de las condiciones, al expresar que los constituyentes de la multiplicidad del mundo objetivo son las condiciones tanto casuales como necesarias, en tanto provocación o engendro necesario de alguna cosa. Los constituyentes de esa multiplicidad son las condiciones heterogéneas y dinámico cambiantes que existen y conforman un conjunto de sistemas interactuantes, en el cual no hay lugar para hechos aislados o encerrados en sí mismos sino que vinculados, aunque su vinculación no sea en relación con todos los aspectos, ni desde siempre o por siempre.

Respecto al conglomerado de condiciones que es llamado causa genética, por parte de los autores originales [cuando se refieren a los momentos en la composición de la causa], señala que no es conveniente llamar causa a algo que aunque necesario es insuficiente para la producción de un efecto. Y propone denominar a tal conglomerado como componente genético de la causa estructural, pues así se enfatiza también que contribuye a su estructuración. Así mismo, propone denominar como componente de suficiencia, a la llamada otra condición necesaria que, según Sagatovski y Antipov, se agrega para constituir la causa estructural. Puesto que favorece la comprensión de que es la interacción del componente genético con el componente de suficiencia la que precisamente constituye al nuevo conglomerado necesario y suficiente para que simultáneamente se produzca el fenómeno, cuya conformación es denominada causa estructural.

Reitera que no se pueden separar las causas de sus efectos o engendros, de lo contrario se deja espacio para considerarlas factores de inicio, puesto que las causas no existen encerradas en sí mismas. Además, su conformación como complejo causa/efecto no agota la realidad y una vez constituida debe existir en un contexto integrado por múltiples condiciones en continuo cambio, entre las cuales la causa, más bien el complejo causa/efecto, es otra condición también cambiante.

Destaca que el mecanismo de acción de la causa o de esa interacción, de lo interno con lo externo, que necesaria y simultáneamente provoca un efecto exis- 
te en la realidad. Este mecanismo de acción de la concreción de la causa, y el tránsito de la causa al efecto, puede comprenderse como una sucesión de formas de movimiento de determinada sustancia, lo cual fortalece la noción de la unidad estructural del proceso.

Refiere [en concordancia con su propuesta de identificar con mayor precisión a los componentes de la causa] que las condiciones etiológicas incluyen tanto las condiciones que constituyen el componente genético como las condiciones que constituyen el componente de suficiencia, que son todos aquellos elementos cuya interacción eventual provoca un determinado efecto. Manifiesta que los factores que constituyen el componente genético, que claramente anteceden a la aparición del efecto, pueden conformar una secuencia dinámica de condiciones necesarias, algunas de las cuales pueden existir y otras incluso pueden haber desaparecido para cuando se concrete la posibilidad real del efecto. Es decir, pueden haber desaparecido para cuando el componente genético así modificado interactúe con el componente de suficiencia, también dinámico, transformable y transformador, del cual al menos una forma estable podría tener precedencia existencial y temporal en relación con el complejo causa/efecto.

Expresa su convicción de que esta interpretación facilita la comprensión del surgimiento, curso y funcionamiento de un proceso. Además, de que es una interpretación versátil que permite reconocer una causa estructural en cada momento susceptible de observación o de separación del continuum representado por la patogénesis o del continuum del desarrollo de cualquier proceso.

Selva Sutter (1997), por otro lado, plantea que una de las principales limitantes que ha tenido el desarrollo conceptual en el área de la salud, es querer explicar el proceso salud/enfermedad de una forma parcializada, ya sea por atribuirle principalidad al componente biológico o al social. Pero propone que esto encuentra la posibilidad de ser superado con la introducción de un concepto unificador, el de la socionaturalidad de los procesos, que facilita el análisis y comprensión de la naturaleza de los fenómenos, particularmente del proceso salud/ enfermedad.

Este concepto, señala, es una respuesta a las preguntas que eran obligatorias de formularse ante la problemática derivada de la disputa entre la biomedicina y la medicina social, e incluso ante el mismo planteamiento de la causalidad estructural. Cuestionamientos referidos a ¿cuán específica y exclusivamente social es lo social? y ¿cuán específica y exclusivamente biológico es lo biológico? o ¿cuán natural es lo natural en realidad?, o puesto de otra manera ¿cuán excluyente de lo natural es lo social y cuán excluyente de lo social es lo natural?, ¿cuán singularizable o encerrado en sí mismo es lo social o lo natural?.

Según su tesis de la socionaturalidad de los procesos, ni siquiera lo mental puede estudiarse y comprenderse sin considerar las interacciones entre lo inorgá- 
nico, lo conocido como orgánico y lo llamado social, así lo dictan los postulados de las ciencias neurológicas. La mente representa, al menos parcialmente, una vasta extensión de funciones o de procesos desarrollados por el cerebro, una esfera de actividad o vasto espectro de la función cerebral determinada, a la vez, por lo genético (socionatural) y por la experiencia (también socionatural), consecuentemente este postulado es la base para superar el nocivo dualismo mente/cuerpo.

Hace ver que la base científica para ello se encuentra, entre otras cosas, en que las células cerebrales desarrollan interconexiones, las que son posibles de desarrollar debido a los procesos epigenéticos que controlan la diferenciación de las células neurales, que se originan del embrión y del medio externo. Las influencias epigenéticas que derivan del embrión incluyen señales intercelulares que consisten en muchos factores difusibles y moléculas de superficie. En tanto que el ambiente externo provee nutrientes, experiencias sensoriales y sociales, así como aprendizajes que median sus efectos a través de canales en la actividad neural, lo cual hace evidente que lo mental es un fenómeno socionatural.

Concluye, por tanto, que la perturbación de lo mental también responde a estímulos y acciones socionaturales. Pues está ampliamente documentado que las emociones y los cambios del humor, son el resultado de la interacción de lo externo (entorno) con la base cerebral, que lleva a una serie de interacciones dentro del individuo y que terminan con la manifestación de los síntomas. Es un hecho que los estresores psicosociales se originan con la participación fundamental de las relaciones humanas.

Selva Sutter (1992a, 1992b) expone, además, que, desde la conceptualización socionatural del proceso salud/enfermedad, la salud es reconocida como una necesidad humana fundamental, y un atributo esencial de la especie humana relacionada a su evolución, con tendencia a la universalidad. En este momento, la salud se encuentra en su forma irrealizada, pero con la potencialidad de hacerla realidad en la medida en que se desarrollen acciones de intervención integrales y simultáneas. Claro que esto pasa por entender que tanto sus condiciones como sus interacciones son esencialmente socionaturales.

La operativización de las necesidades humanas fundamentales, por una parte considera las categorías existenciales de ser, estar, hacer y tener, y por otra parte considera valores o categorías axiológicas como la subsistencia, la protección, el afecto, el entretenimiento, la participación, el ocio, la creatividad, la identidad, la libertad y la salud. Para posibilitar la vivencia o la realización de las necesidades humanas se impone la generación de satisfactores, que son productos socionaturales. Los satisfactores representan formas de ser, estar, hacer y tener relacionadas con estructuras que contribuyen a satisfacción de las necesidades. Son maneras y medios para la satisfacción relativa de las mismas. Estos se modifican marcadamente al ritmo de la historia, y también se diversifican de acuerdo a las culturas y las circunstancias. El esfuerzo conducente a la realiza- 
ción de las necesidades humanas fundamentales, es la premisa indispensable para la concreción de todo proyecto de desarrollo a escala humana (Selva Sutter, 1992a, 1992b, 1997).

La conceptualización original de Sagatovski y Antipov sobre la causa estructural, promete ser más completa en la comprensión de los fenómenos. Las ampliaciones y puntualizaciones de Selva Sutter, al respecto, posibilitan una aplicación más coherente del planteamiento, optimizando así su potencial explicativo. Del mismo modo, las reflexiones de este último autor en relación con la naturaleza de los fenómenos, introduciendo el concepto de la socionaturalidad de los mismos, permite romper con la tradicional principalidad de lo biológico sobre lo social, sin correr el riesgo de que lo social prevalezca sobre lo biológico. De ahí que este enfoque posea un valor relevante para explicar integralmente, lo relativo al origen y naturaleza de los procesos de salud/enfermedad.

Ahora, se puede pasar a dar cuenta de la causa de la depresión, aplicando los conceptos de este planteamiento. Tal como se ha explicado antes, existe un conjunto de elementos que la investigación rigurosa de corte biomédico ha identificado como factores predisponentes o de vulnerabilidad a la depresión. Estos factores incluyen la transmisión genética, la transmisión familiar, las características psicológicas y el estrés. Específicamente, la gente es propensa o proclive a padecer depresión cuando tiene parientes de primer grado con antecedentes de trastornos del humor; cuando durante la nin̄ez sufrió la pérdida de un ser significativo y esa experiencia no se superó conscientemente o favoreció una identidad insegura; cuando su vida ha transcurrido en un medio social poco estimulante o aversivo; cuando le ha caracterizado un patrón de pensamiento negativista $\mathrm{y} / \mathrm{o}$ pesimista; y cuando se sufre el estrés hasta el nivel de agotamiento.

De acuerdo con la interpretación de la causalidad estructural socionatural, todos estos elementos, comprendidos tradicionalmente como factores de susceptibilidad, son fenómenos definitivamente partícipes en los procesos de depresión. En los términos de esta interpretación, estas son las condiciones que configuran al componente genético ${ }^{4}$; es decir, las condiciones que anteceden al aparecimiento de la depresión y que son necesarias, pero insuficientes en sí mismas, para que ello ocurra.

Como ya se ha explicado también, existe un proceso dinámico vinculado al estrés excesivo, que la investigación ha identificado como el evento que precede de manera inmediata a la depresión. Este proceso es resultante de la interacción del estresor con aspectos de la estructura mental del individuo que da una respuesta ante la situación provocadora de tensión, respuesta que no es ajena, por tanto, a los elementos o condiciones del componente genético individual. Específicamente, conduce a que se desencadene una serie de mecanismos adaptativos internos orientados a restaurar el equilibrio y que al fallar en este propósito se provoca el agotamiento de los mismos. 
De acuerdo con la interpretación de la causalidad estructural socionatural, este elemento, comprendido tradicionalmente como el factor disparador o desencadenante, es un fenómeno igualmente partícipe en los procesos de depresión. En los términos de esta interpretación, este proceso representa las condiciones que configuran al componente de suficiencia; es decir, el conglomerado de condiciones derivado de una interacción tal que al presentarse completa la estructuración de la causa, un conglomerado de condiciones ahora necesario y suficiente para provocar el aparecimiento de su efecto, y simultáneamente la depresión es puesta de manifiesto.

Cabe destacar que la transmisión genética; la transmisión familiar; las condiciones sociales; las características psicológicas; el estrés al extremo del agotamiento; la falla en los mecanismos intemos reguladores; y el proceso que constituye al componente de suficiencia, los cuales son identificados como macrocondiciones de la causa estructural de la depresión en general; son eventos dinámico-cambiantes que están presentes al momento de la expresión clínica de esta entidad patológica.

Así mismo, existe una serie de sucesos que la investigación ha identificado como los eventos que concretan o cristalizan la ocurrencia de la depresión. En el plano de la organización funcional de la corteza cerebral, se presenta una alteración en los sistemas que regulan los niveles del humor y de la actividad. En el nivel neuroquímico y fisiológico, se altera el proceso de transmisión de impulsos entre las células nerviosas. También se alteran los sistemas que mantienen el ambiente interno estable o la homeostasis fisiológica. Las dos anomalías antes señaladas mantienen una estrecha interdependencia. Finalmente, se suceden alteraciones de ritmos circadianos del cuerpo — de 24 horas- señalados por la rotación de la tierra.

De acuerdo con la interpretación de la causalidad estructural socionatural, estos eventos configuran un fenómeno que completa el proceso causal de la depresión. En los términos de esta interpretación, este proceso representa el efecto o el fenómeno provocado. Es el mecanismo interno que se desencadena en el interior del individuo y que subyace a los diferentes síntomas que constituyen la manifestación clínica de la depresión.

La interacción causal de la depresión, por tanto, se realiza cuando un individuo caracterizado por poseer antecedentes familiares de depresión; experiencias de decepción no asimiladas; identidad insegura; tendencia al aislamiento; sentimientos de indefensión; y un patrón de pensamiento pesimista; se enfrenta a cambios en el entorno social que implican alteraciones a su vida y lo someten a un estado de estrés. $Y$ ante esas circunstancias, que ponen en funcionamiento una serie de mecanismos adaptativos o de ajuste, se activan sus esquemas mentales de autoderrota, lo que le impide que logre superar la situación y sucumbe a la crisis. Consecuentemente, se genera un deterioro de las conexiones en las 
redes dedicadas a la regulación del humor, llevando a disminuir el metabolismo cerebral en la región frontal izquierda, así como a un aumento en la actividad de la amígdala. Aunado a esto, el ineficiente sistema de circuitos relacionados con los neurotransmisores monoaminos, localizados principalmente en el hipotálamo y sistema límbico, da lugar a que se produzca una respuesta poco intensa en los autoreceptores pre y postsinápticos, por insuficiente presencia de norepinefrina y serotonina a ese nivel. A su vez, el desequilibrio de los ejes hipotalámico-pituitario-adrenal e hipotalámico-pituitario-tiroideo, derivado de la vuelta inoperante del mecanismo de retroalimentación negativa de este sistema regulador, conlleva a la liberación excesiva de cortisol al torrente sanguíneo por parte de la glándula adrenal, así como a la producción de niveles anormalmente bajos de hormona tiroidea. Simultáneamente a todo ello, se presentan las diferentes manifestaciones clínicas de la depresión, tales como el decaimiento en el estado de ánimo, la pérdida del interés y de la capacidad del individuo para disfrutar de sus actividades, la reducción de la energía que conduce a una fatiga creciente y a la disminución de sus actividades, además de otras.

La etiología de la depresión está constituida por todo este conjunto de condiciones que participan directamente en su desarrollo. Estas condiciones son las que se han indicado configuran al componente genético y al componente de suficiencia, y cuyas interacciones conducen necesaria y simultáneamente a su manifestación. La patogénesis comprende los mecanismos de interacción que se producen entre estas diferentes condiciones etiológicas, secuencial y simultáneamente con su manifestación.

Es procedente señalar que el componente de suficiencia, tal como se ha expuesto, ilustra la noción de que la causa de la depresión incluye tanto al evento de provocación como al fenómeno dado (depresión), y que la causa y el efecto son dos facetas del mismo proceso.

En la causa de la depresión, así entendida, las condiciones necesarias y suficientes, que hacen posible su manifestación, con todas sus implicaciones, tienen un carácter socionatural. Ninguna de ellas puede ser explicada al margen de lo biológico, lo psicológico y lo social, y el surgimiento de cada una de ellas en su individualidad está mediatizado por la acción humana. Los aspectos constitucionales, relacionados particularmente con la transmisión genética, que la biomedicina categoriza como biológicos, no serían posibles sin la participación del humano en el proceso de conservación de la especie. Los aspectos relativos a la estructuración psicológica son un reflejo del medio social y cultural, que derivan de un proceso complejo de interacciones entre el cerebro, a través de los órganos de los sentidos, y el entorno. La transmisión familiar está estrechamente relacionada a la educación en el hogar; la decepción no asimilada y la identidad insegura son un resultado de las experiencias de pérdida de un vínculo emocional en la infancia; el aislamiento es promovido por un medio social poco recompensante 
de las actividades del individuo; los sentimientos de indefensión son aprendidos en condiciones sociales aversivas prolongadas sobre las que no se tiene control; el patrón de pensamiento pesimista y/o negativista también es adquirido en aprendizajes tempranos; el estrés es una respuesta ante acontecimientos que ocurren en el entorno y que exigen una adaptación difícil. La respuesta específica a los acontecimientos o situaciones que exigen una adaptación difícil, es producto de un proceso de interacción entre el estresor y la estructuración mental del individuo forjada por la experiencia y el aprendizaje. No se puede ni debe ignorar que las diversas interacciones producidas en el conglomerado llamado causa estructural, al igual que su efecto, son en esencia socionaturales.

\section{Conclusión}

Los aspectos confirmados por los numerosos estudios realizados, permiten derivar la conclusión de que la depresión es la vivencia del individuo de un sentimiento de tristeza profunda y prolongada expresada en su actividad; que representa subjetivamente la ocurrencia de acontecimientos en el conjunto de relaciones sociales en el cual transcurre su vida que le afectan desfavorablemente; a la cual le subyacen una serie de eventos orgánicos. Por tanto, se podría decir que se trata de un fenómeno que constituye un reflejo mental de lo social que se materializa en lo biológico.

Ciertamente, cada uno de esos aspectos, por ser objetos de estudio de diversas disciplinas científicas, puede ser enfatizado o incluso referido en total independencia de los otros. Pero, aun cuando es perfectamente posible y válido efectuar diversas abstracciones de ese individuo relacionadas con ese estado, no significa realmente que, en cada caso, se trate de un fenómeno esencialmente mental, biológico o social. Una cosa es que lo psicológico, lo biológico y lo social existan como objetos de estudio de ciencias separadas y otra, distinta y errónea, es que existan como realidades independientes.

Para quienes, relacionados con los problemas de salud mental o los problemas de salud en general, tienen aprecio por la ciencia y tienen la convicción de que ésta representa una fuente invaluable de grandes beneficios para la sociedad, las declaraciones acerca de que las alteraciones de la salud mental son realmente trastornos del cerebro, resultan en cierto sentido un aliciente. Sobre todo si son hechas por instituciones o personas de renombre en este campo. Pues le restringen el espacio al mentalismo, que en nada contribuye al desarrollo de las disciplinas involucradas y que todavía acecha este ámbito del saber y del quehacer. Pero, en cierto sentido también, tal motivo de optimismo cesa cuando se observa que las posiciones organicistas se han anquilosado y a la vuelta de un tiempo han terminado desempeñando el mismo papel obstructivo del desarrollo científico que las posiciones que han superado. 
A juicio de Pérez Lovelle (1989) la posición privilegiada que ocupa esta concepción obedece a hechos del pasado, cuyo valor no debe dejarse sin ser reconocido. Considera que, en lo que respecta a la biología de las enfermedades, el siglo XIX es el verdadero siglo de oro de la biomedicina, tanto en el aspecto de los descubrimientos fundamentales realizados, como en el aspecto de la solución de importantes problemas prácticos de la lucha contra las enfermedades. La conceptualización biológica de la causalidad de la enfermedad surge en un determinado momento histórico y adquiere un enorme prestigio porque sustituye en gran medida a las explicaciones supersticiosas de la causalidad de la enfermedad. Señala que esto condujo a dos resultados importantes, el primero es que se establece la posibilidad de discriminación precisa de los fenómenos biomédicos, y el segundo, no menos importante, es el hecho de que el propio éxito de la medicina reforzó sensiblemente el punto de vista generalizado de que el biológico es el enfoque científico de la medicina. Hace ver que una consecuencia pemiciosa de esta lamentable situación es que la biomedicina reclama aún la propiedad absoluta del estudio de la salud y de la enfermedad. Sobre la base de una determinación biológica excluye a la sociología y a la psicología, cometiendo el error de abstraer lo biológico del único lugar donde existe concretamente: el individuo.

De ahí que al apreciar, en su justa medida, las razones que llevaron a esta concepción a gozar de la posición de predominio que ocupa hoy en día, se debe admitir que ha sido la cristalización de un progreso histórico de incalculable valor. Pero no se configuró como contribuyente del adelanto científico, sino, por el contrario, se ha configurado como una condición que obstruye nuevos progresos.

Ahora, no debe de pensarse que este es un problema de conceptos únicamente, sino también de prácticas inspiradas o guiadas por esos conceptos. Tal es el caso del tratamiento farmacológico de la depresión, el cual, como ya se ha mencionado, constituye el tratamiento más ampliamente usado. Y no es de extrañar, pues una noción estrecha, acerca de la causa del problema, se concreta en una acción, para su abordaje, igualmente estrecha.

Una evidencia de ello la constituyen algunos índices que ponen en duda la eficacia de la medicación como opción única de tratamiento. Se reconoce que uno de los problemas más desafiantes de la investigación de la depresión y de la práctica clínica es que mientras el $80 \%$, aproximadamente, de gente con depresión responden muy positivamente al tratamiento, un número significativo de individuos no lo hace. Incluso entre los que responden positivamente al tratamiento, muchos no tienen una mejoría completa o duradera y los efectos secundarios adversos son comunes (National Institute of Mental Health, 1999).

Son estos resultados eficazmente débiles los que se derivan de las prácticas limitadas que se inspiran en nociones reduccionistas y es justamente este hecho una expresión concreta de la crisis de la salud pública. 
Por tanto, realmente constituye un reto el logro de una concepción coherentemente científica de los problemas de salud que se corresponda con las necesidades de la sociedad y una tarea que se impone necesariamente es el trabajo sobre los fundamentos conceptuales que prevalecen sobre estos tópicos.

Los hechos, empíricamente confirmados, que en torno a la depresión han sido consignados, evidencian que ésta no sólo puede ser definida como un fenómeno socionatural y con causa estructural sino que son estos términos los que mejor y más fielmente la definen. Por tanto, vuelve realidad la comprensión causal, según la cual no existen causas únicas ni múltiples sino más bien una causa peculiar, estructurada y articulada constituida por diversas condiciones interactuantes que necesaria y simultáneamente producen un efecto.

Entendido así el origen y la naturaleza del problema de salud, se reconoce un nuevo e importante nivel de prevención de la enfermedad: evitar la conformación de los factores o condiciones etiológicas socionaturales de los diversos procesos. Ello implica tener presente el hecho de que la especie humana tiene una clara injerencia en la conformación de la mayoría de las condiciones, en la conformación de los que se conocen como agentes etiológicos o en la conformación de aquellos a los que se identifican como factores de la causa (Selva Sutter, 1992a, 1992b).

La intervención preventiva, orientada y/o dirigida a evitar la producción o generación de condiciones etiológicas y sus interacciones, requiere de un abordaje transdisciplinario del problema. Para el desarrollo de una investigación que conduzca a intervenciones efectivas, desarrolladas simultáneamente y con igual intensidad en todos los niveles en los cuales se puede y debe actuar, con el claro propósito de lograr la salud en su forma realizada. En el nivel individual, acciones tendientes a lograr cambios en el organismo humano para aliviar los síntomas, curar y prevenir la enfermedad y/o cambios que promuevan la salud. En el nivel intermedio, acciones dirigidas a lograr cambios en el ambiente socionatural. Y en el nivel básico, acciones orientadas a lograr cambios en el hecho socionatural general o estructura social, el cual es inseparable de la especie humana (Selva Sutter, 1999).

Esta nueva conceptualización representa enormes ventajas a diferentes niveles. Al trabajador de salud le permite una visión más integral del proceso salud/ enfermedad, dando la pauta para que se convierta en un agente capaz de efectuar un análisis más profundo y completo de dicho fenómeno, facilitando la aplicación de medidas de atención y prevención más integrales, eficaces, eficientes y efectivas. A la población en general le garantiza, de alguna manera, que recibirá una atención que va más allá de la atención médica tradicional, es decir, que las acciones que serán efectuadas estarán también orientadas a impedir no generar las condiciones etiológicas y sus interacciones. A las instituciones formadoras de recursos y dedicadas a la investigación, les provee de un instrumento conceptual 
idóneo que en un futuro pueda ser apreciado y comprendido, y logre ser llevado plenamente a la práctica el abordaje transdisciplinario de los diversos problemas de salud.

Por lo que es conveniente proponer que los profesionales de la salud nos involucremos, desde nuestras posiciones, en la consecución del propósito de superar el análisis desarticulado de los fenómenos relativos al proceso salud/ enfermedad, a través de la estructuración de la causa que provoque la aplicación sistemática de este enfoque. En el entendido que tal posibilidad no se deriva únicamente del enfoque mismo sino de una interacción tal entre éste con un conjunto de condiciones académicas, ideológicas, políticas, sociales y económicas. En ello reside la posibilidad de constatar los beneficios científicos y sociales que es capaz de ofrecer. La invitación es a asumirla como un reto a superar en el futuro.

\section{Referencias bibliográficas}

Alarcón, J. y Kroeger, A. (Eds.) (1991). Taller latinoamericano de epidemiología aplicada a servicios de salud (TLEA). Guia para tutores y participantes. $2^{\mathrm{a}}$ Edición. Lima.

Alleyne, G. A. (1995). "La salud en nuestros dias". En http://www.paho.org/spanish/ opsm9513.html

Andrew, M., McGuffin, P. \& Katz, R. (1998). Genetic and non-genetic subtypes of major depressive disorder. British Journal of Psychiatry, 173:523-526.

Benenson, A. S. (Ed.) (1987). El control de las enfermedades transmisibles en el hombre. $14^{\mathrm{D}}$ Edición. Washington, D.C.: Organización Panamericana de la Salud.

Ferreira, J. R. (1992). La crisis. En Organización Panamericana de la Salud, La crisis de la salud pública: reflexiones para el debate. Washington, D.C.: OPS.

García, J. C. (1994). Las ciencias sociales en medicina. En Organización Panamericana de la Salud, Pensamiento social en salud en américa latina. Washington, D.C.: OPS.

Harvard College (1988). "The nature and causes of depression". The Harvard Medical School Mental Health Letter, March. En http://www.harvardhealthpubs.org/Onlinepubs/ Mental/Mar_1988/index. html

Harvard College (1998). "Mood disorders: an overview". The Harvard Mental Health Letter, January. En http://www. harvardhealthpubs.org/Onlinepubs/Mental/Jan_1998/ index. html

Najera, E. (1992). La salud pública, una teoría para una práctica, se necesita su reconstrucción?. En Organización Panamericana de la Salud, La crisis de la salud pública: reflexiones para el debate. Washington, D.C.: OPS.

National Institute of Mental Health (NIMH) (1999). "Depression research at NIMH". En http://www.nimh.nih.gov/publicat/depresfact.htm

Nemeroff, C. B. (1998). "The neurobiology of depression". Scientific American, June. En http://www.scientificamerican.com/1998/0698issue/ 0698nemeroff.html

Organización Mundial de la Salud (1992). CIE-10. Trastornos mentales y del comportamiento: descripciones clínicas y pautas para el diagnóstico. Madrid: Meditor.

Pardoen, D., Bauwens, F., Dramaix, M., et al. (1996). Life events and primary affective disorders: a one-year prospective study. British Journal of Psychiatry, 169:160-166.

Pérez Lovelle, R. (1989). La psiquis en la determinación de la salud. La Habana: Cientifico-Técnica. 
Roy, A., Nielsen, D., Rylander, G., Sarchiapone, M. \& Segal, N. (1999). Genetics of suicide in depression. Journal of Clinical Psychiatry, 60 Suppl. 2:12-17; discussion 18-20, 113-116.

Sagatovski, V. N. y Antipov, I. G. (1966). Acerca de la correlación entre los conceptos "causa", "condición", "etiología” y "patogénesis". Vestu. Akad. Med. Nauk, SSSR. 21(1):34-40.

Selva Sutter, E. A. (1990). Nuevo concepto sobre el proceso salud-enfermedad. Unidad de Salud Comunitaria, Departamento de Medicina Preventiva y Salud Pública, Facultad de Medicina, Universidad de El Salvador, Mimeo.

Selva Sutter, E. A. (1992a). Lo ineludible de la construcción de un proyecto alternativo para el logro de la salud integral como respuesta a un objetivo del proyecto salvadoreño de nación democrática. Departamento de Medicina Preventiva y Salud Pública, Facultad de Medicina, Universidad de El Salvador, Mimeo.

Selva Sutter, E. A. (1992b). Epidemiología socionatural. Su interacción con un nuevo concepto sobre la calidad de los procesos de salud-enfermedad. Departamento de Medicina Preventiva y Salud Pública, Facultad de Medicina, Universidad de El Salvador, Mimeo.

Selva Sutter, E. A. (1997). Sobre la naturaleza de los Centros de Interacción Social (CIS) y sus implicaciones en los diferentes programas del Departamento de Salud Pública de la UCA (DSP-UCA). Departamento de Salud Pública, Universidad Centroamericana "José Simeón Cañas", Mimeo.

Selva Sutter, E. A. (1999). Más allá de Sagatovski y Antipov: la causalidad estructural puntualizada. Departamento de Salud Pública, Universidad Centroamericana "José Simeón Cañas", Mimeo.

World Health Organization (1997). "The world health report 1997-executive summary. Conquering suffering, enriching humanity". En http://www.who.ch/whr/1997/ exsum97e.html

Zubenko, G. S., Sullivan, P., Nelson, J. P., et al. (1990). Brain imaging abnormalities in mental disorders of late life. Archives of Neurology, 47:1 107-1111.

Zuroff, D. C., Blatt, S. J., Sanislow, C. A., Bondi, C. M. \& Pilkonis, P. A. (1999). Vulnerability to depression: reexamining state dependence and relative stability. Journal of Abnormal Psychology, 108(1):76-89.

\section{Notas}

1. Mantiene una serie de publicaciones periódicas, entre cllas una especializada en el tema de la salud mental.

2. Director del Departamento de Psiquiatría y Ciencias del Comportamiento, en la Escuela de Medicina de la universidad de Emory, y presidente saliente del Colegio Americano de Neuropsicofarmacología.

3. Se declara como la principal organización biomédica de salud mental del mundo. Es uno de los 24 Institutos componentes del Instituto Nacional de Salud (NIH), cl cual pertenece al Departamento de Salud y Servicios Humanos del gobicrno de los EEUU.

4. Con el propósito de evitar cventuales confusiones, cabe reitcrar que tanto Sagatovski y Antipov como Selva Sutter, aplican cl término genćtico para referirse a precedencia en cl tiempo y no a genes o cromosomas. 